\title{
Un análisis empírico de la relación entre el rendimiento de las acciones, el valor económico agregado (EVA) y la utilidad por acción (UPA): Caso México 1998-2012
}

\author{
Julio Téllez Pérez * \\ UNIVERSIDAD ANÁHUAC, MÉXICO
}

\section{Resumen}

El valor económico agregado (EVA) es un medidor financiero que tiene como propósito dimensionar la generación de valor de las empresas, y por su estructura de cálculo, se argumenta que presenta una relación directa al momento de explicar los movimientos de los precios de las acciones $(\mathrm{Rn})$ en comparación con otras medidas financieras contables como la utilidad por acción (UPA). Se tomó una muestra de 58 empresas que cotizaron en la bolsa mexicana de valores entre 1998 y 2012 (15 años), agrupados en tres periodos de 5 años cada uno en función al comportamiento de la economía mexicana. El primero se le denomino periodo de recuperación económica (1), el segundo periodo de crecimiento

(2) y el último periodo de crisis (3). Para encontrar la posible asociación de EVA y UPA con Rn, se utilizó el método generalizado de los momentos (GMM) para las estimaciones, encontrando que UPA presentan la mayor relación con Rn en los periodos económicos (1) y (3) mientras que EVA solo presenta relación significativa en el periodo (2).

Palabras clave: Generación de valor, valor agregado de mercado, valor económico agregado, retorno del capital, flujo libre de efectivo, retorno de los accionistas. Clasificación JEL: C23, C22, D52, D46.

\section{Abstract \\ The Economic Value Added (EVA) is a financial measure that aims to obtain the value creation of companies and for it's fundamentals, it's argued that presents a direct relation in explaining the movements of the prices of Shares $(\mathrm{Rn})$ compared to other accounting measures as the utility per share (EPS). A sample of 58 companies that were listed on the Mexican Stock Exchange over the period 1998 and 2012 (15 years), grouped into three periods of 5 years each in function of the behavior of the Mexican economy. The first was called the post-crisis (1), the second period growth (2) and the last one crisis (3). In order to find the possible association of EVA and UPA with Rn, the generalized Method of Moments (GMM) methodology was used for estimates, finding that UPA have the highest relation with $\mathrm{Rn}$ in the periods (1) and (3) while EVA only present significant relation in the period (2).}

Keywords: Generation of value, market value added, economic value aggregate, return of capital, free cash flow, return of shareholders.

Classification JEL: C23, C22, D52, D46.

jtellez@anahuac.mx* 


\section{INTRODUCCIÓN}

a creación de valor se refiere a la generación de riqueza de una empresa para con sus accionistas a través del aumento del precio de la acción y del pago de dividendos, donde la relación accionista-empresa se da bajo un marco legal y financiero. Mientras que el marco legal proporciona la regulación necesaria para que se efectúe dicha relación, el marco financiero justifica la relación. Por tal motivo, la empresa debe de entender la forma de pensar y de actuar de los accionistas. El comportamiento de los accionistas obedece a un juicio racional: buscar proyectos de inversión que cumplan con las expectativas de retornos esperados. Bajo esta tesitura, la empresa debe demostrar que es un proyecto viable y sostenible en el largo plazo, de otra manera, no podrá atraer capitales para financiar sus proyectos futuros.

El momento crucial que hace que el concepto de creación de valor tome importancia y sea adoptado por las empresas, se da con la publicación del libro Creating Shareholder Value: A guide for Managers and Investors por Alfred Rapapport en 1988, generando una nueva forma de ver a las empresas por parte de los inversionistas y gerentes, bajo el entendimiento que una empresa crea valor cuando el rendimiento de los activos operativos es mayor que el costo de capital que se deriva del financiamiento de los mismos. Con el propósito de medir la creación de valor de las empresas, se han elaborado diversas medidas basadas en una idea en común, la cual es que una empresa crea valor cuando los resultados exceden las expectativas de los accionistas. Cabe mencionar que la gran mayoría de las medidas han sido desarrolladas por empresas de consultoría financiera, como lo son Stern \& Stewart, The Boston Consulting Group, McKinsey, entre otras, desarrollando metodologías que prometen el cálculo y cuantificación de la generación de valor, y que posteriormente las comercializan entre sus carteras de clientes. La insistencia de muchas de estas empresas de consultoría al afirmar que las medidas que proponen son las mejores, obedece al interés monetario que se deriva de la contratación de las consultoras por parte de las empresas, pero no por ello las métricas propuestas carecen de rigor científico y sustento en las principales teorías financieras.

El medidor más utilizado para cuantificar la creación de valor de 
las empresas es la denominada Valor Económico Agregado (EVA por sus siglas en inglés), marca registrada por Stern \& Stewart, y se basa en el concepto de que una empresa debe ganar por lo menos su costo promedio ponderado de capital (WACC por sus siglas en inglés), además que la creación de valor proviene exclusivamente de los resultados operativos. El EVA consiste en determinar el monto que resulta de deducir de la utilidad de operación neta de impuestos, el costo de capital de los recursos propios y externos que utiliza para su operación. Sí el EVA es positivo, significa que la empresa ha generado una rentabilidad por arriba de su costo promedio ponderado de capital, lo que le genera una situación de creación de valor, mientras que si es negativo, se considera que la empresa no es capaz de cubrir su costo promedio ponderado de capital y por lo tanto está destruyendo valor para los accionistas. Independientemente del valor económico agregado, existen otras medidas de amplio uso para cuantificar el desempeño de las empresas como la utilidad por acción (UPA).

El interés por encontrar el medidor más adecuado para dimensionar la creación de valor ha generado una vasta línea de investigación que tiene como propósito determinar la medida más eficiente, y para ello, la mayoría de las investigaciones se enfocan en tratar de encontrar la posible relación entre los precios de las acciones y los medidores de valor.

El presente trabajo de investigación obedece al interés para encontrar el grado de asociación incremental entre medidas financieras basadas en la utilidad por acción (UPA) y el valor económico agregado(EVA) con la rentabilidad de las acciones (Rn).

Laestructura del trabajo de investigación inicia con la introducción, donde se expone de manera conceptual el tema de creación de valor, posteriormente, se hace una revisión de los principales trabajos empíricos que se han realizado al respecto, para continuar con la descripción de la metodología utilizada para encontrar el posible grado de asociación incremental entre la variable dependiente con las independientes seleccionadas en la hipótesis a contrastar, y por último, se analizan los resultados obtenidos, comparando estos con previas investigaciones en esta línea de investigación. 
REVISIÓN DE LA LITERATURA

Ball y Brown (1968) se les considera los precursores de la línea de investigación que tiene como finalidad encontrar la posible relación entre la información contable de las empresas con los precios de las acciones. Dichos autores demostraron que los resultados contables poseen contenido informativo y que afectan a la formación de los precios de las empresas que cotizan en los mercados de capitales. El trabajo de investigación de Ball y Brown motivo a que otros investigadores estudiaran la posible relación entre precios e información contable, destacando los estudios realizados durante los años 90's por Easton y Harris (1991), Ohlson y Penman (1992), Barth (1994), Dechow (1994), Ohlson (1995) y Collins, Maydew y Weiss (1997), donde el común denominador de sus investigaciones era la de encontrar posibles vínculos que pudieran explicar y/o en su caso predecir los precios de las acciones.

El interés por encontrar las posibles relaciones entre el valor de las acciones y la información contable genero una importante línea de investigación que Holthausen y Watts (2001) la denominaron Relevancia del Valor (Value-Relevance en inglés), donde los estudios enfocados a la creación de valor forman parte importante.

Holthausen y Watts (2001) comentan que los trabajos realizados sobre dicha línea de investigación se pueden clasificar en 3 categorías: a) Estudios de asociación relativa.- que son los que comparan la asociación entre el precio de las acciones o el cambio en el precio y medidas financieras basadas en la contabilidad, utilizando el coeficiente de determinación $\left(\mathrm{R}^{2}\right)$. La medida financiera con el mayor $\mathrm{R}^{2}$ se considera que es la que mayor impacto tiene; b) Estudios de asociación incremental.- tienen como propósito investigar sí una medida financiera en particular ayuda a explicar el comportamiento de otras variables específicas. Se considera que una medida financiera es relevante cuando su coeficiente estimado, derivado de una regresión, es significativamente diferente a cero, y c) Contenido de información marginal.- se refiere al estudio de un conjunto de cifras contables específicos si agregan valor a la información disponible para los inversionistas. La metodología que más se usa en este tipo de estudios es el estudio de eventos y tiene como propósito determinar si la nueva información contable generada por la empresa tiene efectos 
en el valor de las acciones. Holthausen et al. (2001) concluyen que el 94\% de los estudios empíricos que tiene que ver con la relevancia del valor, lo hacen bajo la categoría de asociación relativa e incremental mientras que el $6 \%$ restante se realizan bajo estudios de eventos u otro tipo de metodología.

Sharma y Kumar (2010) hacen una revisión de las principales publicaciones, 112 trabajos en total, sobre el EVA entre 1994 y 2008 donde clasifican los trabajos en 7 grupos según la relación que tiene el EVA con: 1) los precios de las acciones, 2) el valor agregado de mercado $\left(\mathrm{MVA}^{1}\right)$, 3) las compensaciones a los gerentes, 4) el proceso de implementación, 5) la administración basada en el valor, 6) valor del dinero a través del tiempo y 7) la revisión de literatura. Los autores encontraron que el $52 \%$ de los trabajos revisados se enfocaron a encontrar una posible relación entre el EVA y los precios de las acciones.

Los trabajos empíricos que más destacan sobre la posible relación del EVA con los precios de la acción son los realizados por: Stewart (1991) estudió la relación entre el EVA y MVA de una muestra de 613 empresas de Estados Unidos entre 1987 y 1988 (2 ańos), encontrando una fuerte relación entre ambas medidas, concluyendo que el EVA es la medida que mejor explica el valor de mercado de las acciones en un $50 \%$ mejor en comparación con medidas tradicionales como el ROE y UPA. O’Byrne (1996) estudió la relación entre los cambios del EVA con las variaciones de los precios de las acciones de una muestra compuesta por 6,551 empresas de Estados Unidos entre 1983 y 1993 (11 ańos), sustentando que el EVA es la variable que mayor relación presenta en los rendimientos de las acciones en comparación con las utilidades al explicar hasta en un $74 \%$ los cambios en el valor de mercado de las empresas. Lehn y Makhija (1997) estudiaron la correlación entre diferentes medidas financieras, los retornos de las acciones y el desempeńo de los directores generales utilizando una muestra de 452 empresas de Estados Unidos durante el periodo 1985-1994 (10 ańos), encontrando que el EVA es el medidor que mayor correlación tiene con el valor de mercado de las empresas en comparación con el retorno de los activos (ROA) y de capital (ROE), además argumentan que el mercado laboral evalúa el desempeño de los directores en función 
al EVA. Bao y Bao (1998) estudiaron la relación del EVA con las utilidades anormales de 166 empresas de Estados Unidos entre 1992 y 1993 (2 años), concluyendo que el EVA es la variable con mayor relevancia al explicar las utilidades anormales en comparación con las utilidades. Machuga, Pfeiffer y Verma (2002) estudiaron la relación entre EVA y UPA de 362 empresas de Estados Unidos en el periodo comprendido entre 1981 y 1996 (16 ańos), afirmando que el EVA contiene información que influye de manera incremental en la UPA al momento de predecir las utilidades de las empresas. Worthington y West (2004) analizaron la relación entre el EVA y los retornos de las acciones de un grupo de 166 empresas australianas entre 1992 y 1998 (7 ańos), obteniendo evidencia que los movimientos en los precios de las acciones están más relacionados con el EVA que con el modelo de utilidades residuales, utilidades y el flujo de caja. Saavedra y Saavedra (2012) estudiaron la relación entre EVA, MVA y la utilidad por acción de 128 empresas mexicanas de 2001 a 2008 (8 años) encontrando que el EVA solo tiene relación con algunos sectores económicos como el de alimentos, comunicaciones y comercio mientras que muestra una relación no significativa con construcción, vivienda y extracción con los retornos de las acciones.

Sin embargo, existe un grupo de trabajos empíricos importante que sugiere que el EVA no presenta una relación significativa con los retornos de las acciones, sobresaliendo el trabajo de Biddle, Bowen y Wallace (1997) que compararon los retornos de las acciones con el EVA, el modelo de utilidad residual y el flujo de efectivo operativo de 773 empresas de Estados Unidos entre 1983 y 1994 (11 años), encontrando que el contenido informativo de las utilidades presentan una mayor relación con los retornos de las acciones. Chen y Dodd (1997) analizaron 605 empresas de Estados Unidos durante el periodo 1983 a 1992 (10 ańos) encontrando que el valor económico agregado no tiene un mayor contenido de información que las medidas tradicionales que se derivan de la utilidad contable, adicionalmente encontraron que el EVA y el modelo de Utilidad Residual (Ohlson 1995) dan los mismos resultados al momento de explicar los retornos de las acciones. Fernández (2002) analizó la correlación del EVA y UPA con la creación de valor de los accionistas de 582 empresas de Estados Unidos y 28 empresas de España 
durante 1991 a 1998 (8 años) encontrando un mínimo grado de correlación por parte del EVA y una mayor correlación de la UPA. Copeland, Dolgoff y Moel (2004) investigaron la relación entre las expectativas de los retornos, EVA y UPA de 499 empresas de Estados Unidos entre 1992 y 1998 (6 años) encontrando que la UPA presenta mayor relación en comparación que el EVA. Ismail (2006) estudio la relación entre EVA y el retorno de las acciones de 281 empresas del reino unido de 1990 a 1997 (8 años), encontrando una relación no significativa del EVA con respecto a las variaciones en los precios de las acciones y que los medidores tradicionales basados en las utilidades mostraban una significativa relación.

\section{METODOLOGÍA Y VARIABLES}

Asumiendo que el retorno de las acciones ( $R n)$ se ve afectado por la diferencia entre la información contenida en los valores realizados de los medidores contables $\left(\mathrm{X}_{\mathrm{t}}\right)$ y las expectativas de mercado $\mathrm{E}(\mathrm{X})$, se tiene el siguiente modelo:

$R n_{i t}=b_{0}+b_{1}\left[X_{i t}-E\left(X_{i t}\right)\right]+\mu_{i t}$

donde $\mathrm{i}=1, \ldots, \mathrm{N}$ y $\mathrm{t}=1, \ldots \mathrm{T}$, considerando $\mathrm{i}$ como la unidad de estudio (corte transversal) y t como la dimensión de tiempo, Rn es la variable dependiente, $b_{0}$ y $b_{1}$ son los vectores de los coeficientes y $[X i t-E(X i t)]$ es la información inesperada contenida en el medidor contable y $\mu$ son los errores aleatorios.

Considerando que las expectativas de mercado $E\left(X_{i}\right)$ se comportan como un proceso estocástico lineal discreto (Biddle, Seow y Siegel 1995):

$E\left(X_{i t}\right)=\gamma+\partial_{1} X_{i t-1}+\partial_{2} X_{i t-2}+\partial_{3} X_{i t-3}+\ldots+\partial_{n} X_{i t-n}$

donde $\gamma$ es una constante y $\partial$ son los parámetros autorregresivos, sustituyendo (2) en (1) obtenemos:

$R n_{t}=b_{0}+b_{1}\left[X_{i t}-\left(\gamma+\partial_{1} X_{i t-1}+\partial_{2} X_{i t-2}+\partial_{3} X_{i t-3}+\ldots+\partial_{n} X_{i t-n}\right)\right]+$ $\mu_{i t}$

Por lo tanto, el comportamiento de los rendimientos de las acciones se ve afectado solamente por la información no esperada, que no se observa de manera directa, del medidor contable en un momento determinado en el tiempo.

Para evitar los problemas que se originan de los cambios estructurales a través del tiempo (Biddle at el. 1995), solo se 
considera un periodo de retardo en $E\left(X_{i t}\right)$, y para mitigar los posibles problemas de escala y heterocedasticidad (Easton, 1999; Chen y Dood, 2001), las variables $\left(\mathrm{X}_{\mathrm{it}}\right)$ y $E\left(X_{i t}\right)$ se deflactaron utilizando el valor de mercado al 31 de diciembre del año $t-1$, generando el siguiente modelo:

$R n_{t}=b_{0}+b_{1} X_{i t} / P_{t-1}+b_{2} X_{i t-1} / P_{t-1}+\mu_{i t}$

Como el propósito de la presente investigación es determinar si el nivel de información que proporciona el medidor EVA afecta de mayor manera a los comportamientos de los precios de las acciones con respecto a la información que contiene la UPA, es necesario realizar un análisis de información incremental, evaluando la significancia de la pendiente de los coeficientes de las dos variables de estudio:

$$
\begin{aligned}
& R n_{t}=b_{0}+\beta_{1} U P A_{t} / P_{t-1}+\beta_{2} U P A_{t-1} / P_{t-1}+\beta_{3} E V A_{t} / P_{t-1}+ \\
& \beta_{4} E V A_{t-1} / P_{t-1}+\mu_{i t}
\end{aligned}
$$

Para complementar el análisis del grado de asociación incremental que pudieran tener las variables independientes con la variable dependiente, se aplicó el estadístico F para rechazar o aceptar la siguiente hipótesis nula (restricción):

$H_{0 k}: \beta_{k t}=\beta_{k t-1}=0$

$H_{A k}: \beta_{k t}=\beta_{k t-1} \neq 0$

Sin embargo, al momento de estimar la ecuación (5) surgen dos problemas econométricos que son necesarios resolver. El primero es la posible relación bidireccional que existe entre la UPA y EVA (endogeneidad) haciendo que ambos medidores estén relacionados con los errores (Baltagi 2005), y el segundo problema proviene de las características invariantes en el tiempo (efectos fijos) por individuo que se encuentran en los errores aleatorios $\left(\mu_{i t}\right)$ de la ecuación (1) y que podrían estar relacionados con las variables independientes: $\mu_{i t} \stackrel{=}{=} v_{i t}+\varepsilon_{i t}$

donde $v_{i t \text { son }}$ los efectos no observables derivados de las características individuales de los individuos y $\varepsilon_{i t}$ representa los errores específicos.

Para lidiar con ambos problemas, se construyó un modelo dinámico utilizando el método generalizado de los momentos (GMM) que usa variables instrumentales basadas en las primeras diferencias de los regresores (Arellano y Bond 1995, Blundell y Bond 1998) y variables proxys, transformando la ecuación (5) y 
agregando la variable de control (variable exógena), tenemos que:

$R n_{i t}=B_{0} R n_{i t-1}+\beta_{1} U P A_{t} / P_{t-1}+\beta_{2} U P A_{t-1} / P_{t-1}+\beta_{3} E V A_{t} / P_{t-1}+$

$\beta_{4} E V A_{t-1} / P_{t-1}+\operatorname{Igae}_{i t}+\varepsilon_{i t}$

donde $R n_{i t}$ son los retornos de las acciones contemporáneos, $R n_{i t-1}$ es su variable rezagada, UPA representa la utilidad por acción, EVA es el valor económico agregado, Igae es el índice global de la actividad económica de México y $\varepsilon$ es el error específico. Adicionalmente se considera la matriz de pesos

heterocedásticos para la estimación (two-step) haciendo que los estimadores sean más eficientes (Roodman 2009) en comparación con los que se obtienen utilizando solamente la matriz de pesos homocedásticos (one step).

Mediante la estimación de la ecuación (6), se busca contrastar la siguiente hipótesis:

$H_{1:}$ El valor económico agregado (EVA) es la mejor medida financiera para explicar el rendimiento de las acciones $(R n)$ en comparación con la utilidad por acción (UPA).

\section{VARIABLES}

A continuación se describen las variables que integran el modelo de relación causal expresado en la ecuación (6).

Variable dependiente:

El retorno de las acciones $\left(\mathrm{Rn}_{\mathrm{it}}\right)$ se calcula como sigue:

$$
R n_{i t}=\frac{\prod_{t=1}^{12}\left(1+\mathrm{r}_{\mathrm{i}, \mathrm{t}}\right)}{\prod_{t=1}^{12}\left(1+\mathrm{r}_{\mathrm{m}, \mathrm{t}}\right)}
$$

donde $r_{i, t}$ es el rendimiento anual continuo de la acción y $r_{m, t}$ representa el rendimiento anual continuo del mercado de capitales mexicanos medido con el índice de retorno total (IRT), el cual considera la variación del precio de la acción y dividendos de un portafolio de acciones representativas del mercado de capitales mexicanos por 58 empresas. Fama (1998) comenta que el retorno total de los accionistas ajustado por el mercado presenta menos sesgo que la diferencia del promedio entre los retornos acumulados de la empresa y el mercado de capitales en el largo plazo. Ambos rendimientos consideran un periodo de 12 meses que termina dos 
meses después del cierre fiscal anual, lo anterior permite que los precios de las acciones reflejen la información contenida en el último reporte financiero emitido por las empresas.

Variables Independientes:

El valor económico agregado $\left(\mathrm{EVA}_{\mathrm{it}}\right)$ se calcula utilizando la fórmula propuesta por Stern \& Stewart:

$\mathrm{EVA}_{\mathrm{it}}=\mathrm{UNO}_{\mathrm{it}}-\mathrm{Cargo}$ por uso de Capital ${ }_{\mathrm{it}}$ donde UNO es la utilidad neta de operación que representa la utilidad de operación después de impuestos y Cargo por uso de Capital representa el costo de los activos involucrados en la generación de la utilidad de operación, que se obtiene de la multiplicación de la inversión de capital en los activos netos (AN) con el costo promedio ponderado de capital (WACC por sus siglas en inglés).

La utilidad por acción se obtiene:

$\mathrm{UPA}_{\mathrm{t}}=\mathrm{UN}_{\mathrm{t}} / \mathrm{No}^{\circ}$ de Acciones $_{\mathrm{t}}$

donde UN es la utilidad neta antes de partidas extraordinarias y operaciones discontinuas.

El Índice Global de Actividad Económica (Igae) es un índice que calcula el Instituto Nacional de Estadística y Geografía (INEGI) de México cuyo propósito es medir la generación de riqueza de México en un periodo determinado.

\section{DESCRIPCIÓN DE LA MUESTRA}

La investigación empírica se realizó con $58(\mathrm{n}=58)$ empresas de la Bolsa Mexicana de Valores (BMV) que cotizaron durante el período 1998 a 2012 ( $\mathrm{t}=15$ años), excluyendo empresas financieras. La fuente donde se obtuvo la información en su totalidad proviene del Economatica. La tabla 1 muestra la descripción de las variables destacando el promedio del EVA-que es negativo, infiriendo que las empresas no generaron valor a los accionistas durante el periodo de análisis, sin embargo, no es un resultado concluyente debido a que el promedio es muy sensible a los valores extremos, aunado que considera el valor económico agregado total en partes iguales entre cada observación, haciendo que el resultado que registra no sea representativo de la muestra. El promedio de $\mathrm{R}(\mathrm{n})$ es cercano a 1 , indicando que el rendimiento promedio de las empresas es similar 
al rendimiento promedio mostrado por el mercado de capitales mexicanos durante el periodo de análisis, infiriendo que el mercado de capitales de México es, de cierta manera, un mercado eficiente al cumplir con uno de los principios de la línea de mercado de valores, el cuál estípula que el rendimiento esperado de un activo financiero debe ser igual al rendimiento esperado del mercado en el tiempo (Sharpe 1964, Linther 1965). La UPA registra un promedio positivo de 0.0898 , sin embargo es la variable que mayor heterogeneidad presenta en comparación con el EVA al mostrar un coeficiente de variación de 23.3419. El promedio del Igae es de 91.94 con un coeficiente de variación de 0.0993 , siendo la variable con menor volatilidad.

\section{Tabla 1}

Descripción de las variables

\begin{tabular}{cccccc} 
Variable & Promedio & $\begin{array}{c}\text { Desviación } \\
\text { Estándar }\end{array}$ & Mínimo & Máximo & $\begin{array}{c}\text { Coeficiente } \\
\text { de variación }\end{array}$ \\
\hline $\mathrm{R}(\mathrm{n})$ & 0.9941 & 0.3371 & 0.8453 & 1.1332 & 0.3391 \\
$\mathrm{UPA}^{*}$ & 0.0898 & 2.0961 & -14.111 & 51.2197 & 23.3419 \\
$\mathrm{EVA}^{-}$ & -0.1310 & 1.1358 & -28.992 & 10.3271 & -8.6702 \\
Igae & 91.94 & 9.13 & 77.200 & 108.500 & 0.0993 \\
\hline
\end{tabular}

*Deflactado utilizando el precio de la acción al 31 de diciembre del año t-1, -Deflactado utilizando el valor de mercado al 31 de diciembre del ańo t-1 para mitigar posibles problemas de escala y heterocedasticidad (Eaton, 1998; Chen y Dood, 2001).

La tabla 2 muestra la correlación de Pearson de manera matricial, donde la variable Igae es la que mayor relación lineal presenta con $\mathrm{R}(\mathrm{n})$ al registrar un coeficiente de correlación positivo de 0.1698 . Caso contrario con UPA y EVA, ya que ambas muestran un grado mínimo de asociación con el retorno total ajustado por el mercado al generar coeficientes de correlación de 0.0399 y 0.0680 respectivamente. Lo anterior indica que las variables que afectan a la creación de riqueza de la economía mexicana influyen de manera directa en la formación de precios de la acciones (efectos externos) en comparación con la situación interna de las empresas reflejada a 
través de la UPA y EVA.

Tabla 2

Matriz de Correlación de Pearson

(R) 1998 - 2012

\begin{tabular}{rcccc}
\hline & $\mathrm{R}(\mathrm{n})$ & $\mathrm{UPA}^{*}$ & EVA $^{-}$ & Igae \\
\hline $\mathrm{R}(\mathrm{n})$ & 1 & & & \\
UPA $^{*}$ & 0.0399 & 1 & & \\
EVA $^{-}$ & 0.0680 & 0.0426 & 1 & \\
Igae & 0.1698 & -0.0187 & 0.0584 & 1 \\
\hline
\end{tabular}

*Deflactado utilizando el precio de la acción al 31 de diciembre del año t-1,

-Deflactado utilizando el valor de mercado al 31 de diciembre del año t-1.

La gráfica 1 muestra la evolución histórica del Igae durante los 15 años analizados mostrando un crecimiento anual compuesto de $2.295 \%$. Diversos han sido los factores que han sucedido en este periodo de tiempo, siendo la más relevante la crisis mexicana de 1995 que afectó el sistema financiero nacional, en especial al sector bancario, obligando al gobierno federal a implementar medidas drásticas de control. A partir de 1996, empiezan a surtir efecto las nuevas políticas económicas implementadas logrando revertir la precaria situación económica del país y es a partir del 2003 cuando los fundamentales de la economía mexicana se estabilizan, y en consecuencia, se genera un importante crecimiento que dura hasta 2007. La crisis global del 2008 impacta de manera negativa al desempeño económico de México provocando una caída del $4.7 \%$ de la creación de riqueza del país para 2009, retomando el crecimiento en 2010 en adelante. 


\section{Gráfica 1}

Índice Global de Actividad Económica (Igae) 1998 - 2012

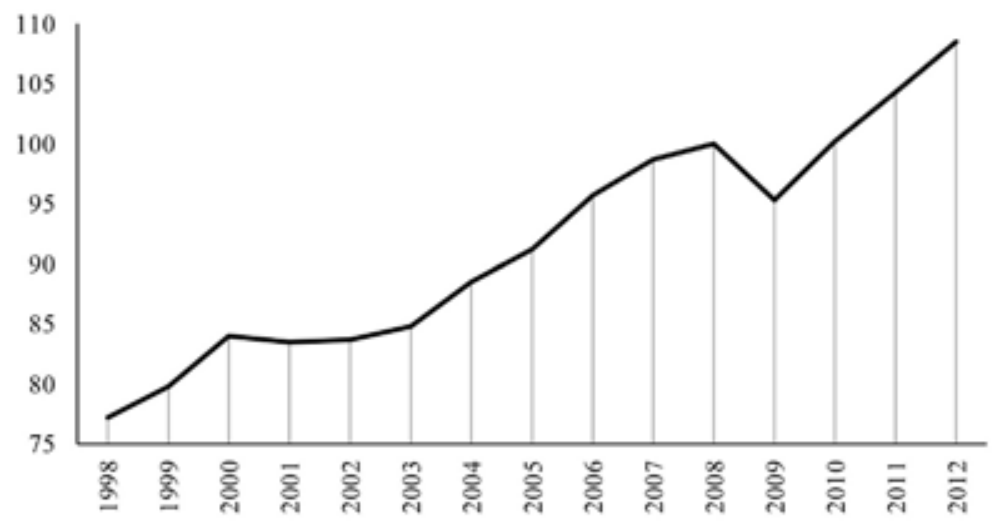

Fuente: Elaboración propia con datos del INEGI

\section{REsultados EMPÍRICOS}

La tabla 3 muestra que $\mathrm{EVA}_{\mathrm{it}}$ y $\mathrm{EVA}_{\mathrm{it}-1}$ presentan un mayor estadístico $\mathrm{F}$ con respecto al registrado por la UPA $\mathrm{ite}_{\mathrm{it}-1}$, indicando que en términos relativos, el EVA es la variable que mayor cantidad de información aporta al explicar los cambios en Rn durante el periodo de análisis 1998 a 2012. Lo anterior va en línea con los resultados mostrados en previas investigaciones (Stewart 1991, Lehn y Mackhijia 1997, Bao y Bao 1998, Feltham et al. 2004, Worthington y West 2004) donde concluyen que el valor económico agregado es la métrica financiera que mayor asociación presenta con los retornos de las acciones en comparación con las medidas tradicionales de desempeño basadas en los resultados contables. 
Tabla 3

Método generalizado de los momentos (GMM)

$$
1998-2012
$$

Observaciones: 843, Grupos: 58, Instrumentos 50

Hansen Test ${ }^{\mathrm{a}}>\mathrm{chi}^{2}$ : (0.185), AR(2): (0.353)

\begin{tabular}{rrrrrr} 
& UPA $_{\mathrm{it}}$ & $\mathrm{UPA}_{\mathrm{it}-1}$ & EVA $_{\mathrm{it}}$ & EVA $_{\mathrm{it}-1}$ & \multicolumn{1}{c}{ Igae $_{\mathrm{it}}$} \\
\hline Coeficiente & .0000 & 0.0003 & 0.0024 & -0.0026 & 0.0007 \\
& 1.55 & 0.5383 & 30.47 & -110.46 & 57.00 \\
Estadístico $t$ & $(0.127)$ & $0.000)^{*}$ & $(0.000)^{*}$ & $(0.000)^{*}$ & $(0.000)^{*}$ \\
& 694.5 & & & 6377.60 & \\
Estadístico $F$ & $(0.000)^{*}$ & & & $(0.000)^{*}$ & \\
valor $p^{a}$ & & & & \\
\hline
\end{tabular}

${ }^{* * *}$ significativo al $10 \% ;{ }^{* *}$ significativo al $5 \%$; ${ }^{*}$ significativo al $1 \%$

a Prueba estadística de la hipótesis nula de las restricciones de sobreidentifación son válidas (two step).

${ }^{b}$ El valor $\mathrm{p}$ en paréntesis representa la prueba estadística de dos colas de la hipótesis nula de no contenido de información incremental, Ho: $\beta_{\mathrm{it}}=\beta_{\mathrm{it}-1}=0$.

Cabe destacar que la variable exógena $\operatorname{Igae}_{\text {it }}$ es la que mayor incidencia tiene en los movimientos en los precios de la acción, infiriendo que los movimientos de las acciones en el mercado de valores presenta una relación lineal directa con la actividad económica del país.

Para corroborar los resultados anteriores, se segmento el horizonte de análisis en 3 periodos de tiempo: el primero abarca 1998 a 2002, el segundo 2003 a 2007 y el último 2008 a 2012. El criterio que sé utilizo para dicha segmentación fue la situación económica de México. El primer grupo se considera como un periodo de postcrisis, el segundo periodo es de crecimiento y el último grupo es de crisis. Lo anterior permite ver si la relación casual del EVA con respecto a $\mathrm{Rn}$ es persistente cuando se presentan distintos fenómenos económicos que afectan a un país.

La tabla 4 panel a muestra los resultados del primer grupo 1998 - 2002 mostrando que la UPA es la variable que mayor incidencia tiene con Rn en comparación con el EVA al mostrar un estadístico $\mathrm{F}$ de

85.03. El panel b muestra que para el periodo de tiempo 2003 
- 2007 EVA es la variable que más incide al explicar la variación en los precios de las acciones que UPA al mostrar un estadístico $F$ de 189.96. El panel c enseña los resultados obtenidos del último periodo analizado 2008 - 2012 donde al variable UPA es la variable que mayor relación tiene al momento de explicar las variaciones en Rn en comparación con EVA al registrar un estadístico F de 917.21 en comparación por el registrado por EVA de 411.76.

\section{Tabla 4}

Método generalizado de los momentos (GMM)

Panel a: 1998 - 2002

Observaciones: 267, Grupos: 55, Instrumentos 20

Hansen Test $^{\mathrm{a}}>\mathrm{chi}^{2}:(0.594), \operatorname{AR}(2):(0.828)$

\begin{tabular}{|c|c|c|c|c|c|}
\hline & $\mathrm{UPA}_{\mathrm{it}}$ & $\mathrm{UPA}_{\mathrm{it}-1}$ & $\mathrm{EVA}_{\mathrm{it}}$ & $\mathrm{EVA}_{\mathrm{it}-1}$ & $\operatorname{Igae}_{\text {it }}$ \\
\hline Coeficiente & .0010 & -.0004 & -.0080 & .0089 & 0.0030 \\
\hline Estadístico t & $\begin{array}{r}7.97 \\
(0.000)^{*}\end{array}$ & $\begin{array}{r}-5.11 \\
0.000)^{*}\end{array}$ & $\begin{array}{r}-2.63 \\
(0.011)^{* *}\end{array}$ & $\begin{array}{r}5.46 \\
(0.000)^{*}\end{array}$ & $\begin{array}{r}7.21 \\
(0.000)^{*}\end{array}$ \\
\hline $\begin{array}{r}\text { Estadístico F } \\
\text { valor } \mathrm{p}^{\mathrm{a}}\end{array}$ & $\begin{array}{r}85.03 \\
(0.000)^{*}\end{array}$ & \multicolumn{4}{|c|}{$\begin{array}{c}13.32 \\
(0.001)^{*}\end{array}$} \\
\hline
\end{tabular}

Panel b: $2003-2007$

Observaciones: 286, Grupos: 58, Instrumentos 35

Hansen Test ${ }^{2}>$ chi $^{2}:(0.359), \operatorname{AR}(2):(0.769)$

\begin{tabular}{rrrrrr}
\hline Coeficiente & -.0008 & .0014 & -.0000 & -.0035 & 0.0005 \\
& -3.21 & 03.55 & -0.14 & -33.15 & 8.43 \\
Estadístico t & $(0.002)^{*}$ & $(0.001)^{*}$ & $(0.889)$ & $(0.000)^{*}$ & $(0.000)^{*}$ \\
& 21.22 & \multicolumn{5}{c}{189.96} \\
Estadístico F & $(0.000)^{*}$ & \multicolumn{5}{c}{$(0.000)^{*}$} \\
valor $\mathrm{p}^{\mathrm{a}}$ & $(0.007$ & \\
\hline
\end{tabular}

Panel c: $2008-2012$

Observaciones: 290, Grupos: 58, Instrumentos 50

Hansen Test ${ }^{\mathrm{a}}>\mathrm{chi}^{2}$ : (0.187), AR(2): (0.481)

$\begin{array}{llllll}\text { Coeficiente } & 0.0670 & 0.0193 & -.0538 & 0.0085 & 0.0015\end{array}$




\begin{tabular}{rrrrrr} 
Estadístico t & 55.53 & 20.84 & -25.39 & 5.96 & 53.08 \\
& $(0.000)^{*}$ & $(0.000)^{*}$ & $(0.000)^{*}$ & $(0.000)^{*}$ & $(0.000)^{*}$ \\
Estadístico F & 917.21 & & & 411.76 & \\
valor $\mathrm{p}^{\mathrm{a}}$ & $(0.000)^{*}$ & & & $(0.000)^{*}$ & \\
\hline
\end{tabular}

***significativo al $10 \%$; ${ }^{* *}$ significativo al $5 \%$; ${ }^{*}$ significativo al $1 \%$

${ }^{\text {a} P r u e b a ~ e s t a d i ́ s t i c a ~ d e ~ l a ~ h i p o ́ t e s i s ~ n u l a ~ d e ~ l a s ~ r e s t r i c c i o n e s ~ d e ~ s o b r e ~ i d e n t i f i c a c i o ́ n ~ s o n ~}$ válidas (two step).

${ }^{\mathrm{b}} \mathrm{El}$ valor $\mathrm{p}$ en paréntesis representa la prueba estadística de dos colas de la hipótesis nula de no contenido de información incremental, Ho: $\beta_{\mathrm{it}}=\beta_{\mathrm{it}-1}=0$.

Al segmentar el periodo de análisis y someter la relevancia y persistencia del indicador EVA a distintos efectos económicos, se puede observar que pierde relevancia en periodos de volatilidad y de no crecimiento económico y cobra relevancia en periodos de estabilidad económica.

\section{CONCLUSIONES}

La evidencia muestra que UPA presentan una relación significativa con el retorno de las acciones en los periodos económicos postcrisis (1998-2003) y crisis (2008-2012), infiriendo que la generación de utilidad neta es la variable más incide al momento de explicar el retorno de las acciones, sin embargo no se le puede considerar como una métrica que cuantifique la creación de valor debido a que su metodología de cálculo no involucra el costo de los recursos financieros, por lo que no hay manera de saber si los resultados mostrados por dicho medidor indica que se está maximizando el valor de los accionistas al no contar con una referencia de comparación.

La hipótesis a contrastar por la presente investigación se rechaza parciamente ya que el EVA no presenta persistencia en explicar las variaciones de los precios de las acciones ya que solo presenta relevancia cuando la volatilidad económica es baja, caso contrario en los periodos económicos de elevada volatilidad.

Este trabajo empírico pretende contribuir a la línea de investigación que estudia la relación entre las medidas de generación de valor con el valor de los accionistas, además de proporcionar información relevante a las empresas que están interesadas en adoptar el medidor EVA como la medida principal de creación de valor en 
sustitución de las medidas contables tradicionales para la toma de decisiones. Creemos que la información y los resultados del presente trabajo contribuyen de manera importante para entender el tema de generación de valor en México, donde la línea de investigación formal sobre este tópico es muy pobre.

Como una posible línea futura de investigación sería la de explorar el costo de capital en México dando énfasis en el estudio del riesgo sistemático y el premio al riesgo del mercado de valores a los inversionistas, así como también explorar otros factores que influyen en la creación de valor como el nivel de experiencia y preparación de la gerencia, estrategia de negocios, problemas de agencias, nivel de eficiencia, tecnología, entre otros.

Bibliografía

ARELlaNO, M. Y BOND, S.R. (1991). Some Tests of Specification for Panel Data: Monte Carlo Evidence and an Application to Employment Equations, Review of Economic Studies, 58, 277-297.

BALL, R. Y BROWN P. (1968). An Empirical Evaluation of Accounting Income Numbers. Journal of Accounting Research, 6, 159-178.

BALTAGI, H.B. (2005). Econometric Analysis of Panel Data ( 3 rd ed.). New York: John Wiley \& Sons.

BARTH, M.E. (1994). Fair value accounting: Evidence from investment securities and the market valuation of banks. Accounting Review, 69, 1-25.

BAO, B.H. Y BAO, D.H. (1998). Usefulness of value Added and Abnormal Economic Earnings: An Empirical examination. Journal of Business Finance and Accounting, 25(1-2), 251265.

BIDDLE, G.C., BOWEN, M.R. Y WALLACE, J.S. (1997). Does EVA beat Earnings? - Evidence on Associations with Stock Returns and Firm Values. Journal of Accounting and Economics, 24(3), 301-336.

BLUNDELL, R. Y BOND, S. (1998). Initial conditions and moments restrictions in dynamic panel data models, Journal of Econometrics, 87, 115-143. 
CHEN, S. AND DODD, J.L. (1997). Economic Value Added (EVA): An Empirical Examination of a New Corporate Performance Measure. Journal of Managerial Issues. 9(3), 318333.

COLLINS, D.W., MAYDEW, E.L. YWEISS, I.S. (1997). Changes in the value-relevance of earnings and book values over the past forty years. Journal of Accounting and Economics, 24(1), 3967.

COPELAND, T., DOLGOFF, A. Y MOEL, A. (2004). The Role of Expectations in the Cross-Section of Returns, Review of Accounting Studies, 9, Issue 2-3.

DECHOW, P.M. (1994). Accounting earnings and cash flows as measures of firm performance: The role of accounting accruals. Journal of Accounting and Economics, 18, 3 - 42.

EASTON, P. D., Y HARRIS, T. S. (1991). Earnings as an Explanatory Variable for Returns. Journal of Accounting Research, (Spring), 19-36.

EASTON, P. D. (1999). Security Returns and the Value Relevance of Accounting Data. Accounting Horizons, 13(4), 399-412.

FAMA, E. (1998), Market Efficiency, Long-Term Returns, and Behavioral Finance. Journal of Financial Economics, 49, 283306.

FERNANDEZ, P. (2002). EVA, Economic Profit and Cash Value Added Do not measure Sharholder Value (Working Paper No. 453). España: IESE - University of Navarra.

FELTHAM, G., ISAAC, G., MBAGWN, C. YVAIDYANATHAN, G. (2004). Perhaps EVA Does Beat Earnings - Revisiting Previous Evidence. Journal of Applied Corporate Finance, 16(1), 83-88.

HOLTHAUSEN, W., WATTS, R. Y ROSS, L. (2001). The relevance of the value-relevance literature for financial accounting standard setting. Journal of Accounting and Economics, 31, 3-75.

ISMAIL, A. (2006). Is EVA associated with stock return than accounting earnings? The UK evidence. International Journal of Managerial Finance, 2(4), 343-353.

LEHN, K. Y MAKHIJA, A.K. (1997). EVA, Accounting profits, and 
CEO turnover: an empirical examination 1985-1994. Journal of Applied Corporate Finance, 10(2), 90-96.

LINTNER, J. (1965). The Valuation of Risk Assets and Selection of Risky Investments in Stock Portfolios and Capital Budgets. Review of Economics and Statistics, 47, 13-37.

MACHUGA, S., PFEIFFER, J Y VERMA, K. (2002), Economic Value Added, Future Accounting Earnings, and Financial Analysts' Earnings Per Share Forecasts. Review of Quantitative Finance and Accounting, 18, 59-73.

O'BYRNE, S.F. (1996). EVA and Market Value. Journal of Applied Corporate Finance, 9(1), 116-125. Ohlson, J. (1995). Earnings, book values, and dividends in equity valuation. Contemporary Accounting Research, 11, 661-687.

OHLSON, J. AND PENMAN, S.H. (1992). Disaggregated Accounting Data as Explanatory Variables of Retuns. Journal of Accounting, Auditing and Finance, 7, 553-573.

RAPPAPORT, ALFRED (1998). Creating Shareholder Value: The New Standard for Business Perfomance $\left(1^{\text {st }}\right.$ ed.). New York: Free Press.

ROODMAN, D. (2009). How to do xtabond2: An Introduction to difference and system GMM in stata, The Stata Journal, 9(1), 86-136.

SAAVEDRA, M.L. Y SAAVEDRA M.J. (2012). El Valor Económico Agregado y su relación con el valor agregado de mercado, la utilidad por acción y el rendimiento de los activos, en México: 2001-2008, Revue Recherches en Sciences de Gestion-Management Science-Ciencias de Gestión, 90, p. 19 a 40.

SHARMA, A.K. Y KUMAR, S. (2010). Economic Value Added (EVA) - Literature review and relevant issues. International Journal of Economics and Finance, 2, 200-220.

SHARPE, W.F. (1964), Capital Asset Prices: A Theory of Market Equilibrium under Conditions of Risk. Journal of Finance, 19, 425-442.

STEWART, G. BENETT (1991). The Quest for Value ( $1^{\text {st }}$ ed.). New York: Harper Collins.

STEWART, G. BENETT (1995, 1 de Mayo). EVA Works But Not 
if you Make These Common Mistakes. Fortune, 117-118. WORTHINGTON, A.C. YT. WEST, (2004). Australian Evidence Concerning the information Content of Economic Value Added. Australian Journal of Management. 29(2)2, 201-223.

Notas de Página

${ }^{1}$ MVA es la diferencia entre el valor de mercado y contable de la empresa. Sí el MVA es positivo, la empresa genero valor, caso contrario, la empresa genero una pérdida en el patrimonio de los accionistas.

Fecha de Recepción del Artículo: 18 de enero de 2017 Fecha de Aceptación: 30 de marzo de 2017 